\title{
CFD analysis of probe interaction with expanding wet steam
}

\author{
Michal Kolovratník*, Gukchol Jun, Ondřej Bartoš \\ CTU in Prague, Department of Energy Engineering, Technická 4, Prague 6, 166 07, Czech Republic.
}

\begin{abstract}
Research on the impact of the interaction between an optical probe and the expanding steam flow field in a steam turbine on the measured parameters of the liquid phase of the wet steam has been ongoing at Czech Technical University in Prague (CTU) for several years. Previous CFD analyses were newly supplemented by the effect of changing the diameter and number of fine droplets as well as changing of steam wetness in the probe measuring space. The knowledge obtained was used to improve corrections of the extinction measurements at CTU and for conceptual design of a new extinction probe for phase transition investigation in high pressure steam. This paper briefly introduces these results.
\end{abstract}

\section{Introduction}

Experimental research of phase transitions in wet steam expanding in turbines is typically based on optical extinction probes. These probes allow for the measurement of steam wetness and the diameters of fine droplets within the measuring space of the probe, i.e. locally in relation to the position of the probe. Repeated measurement in various points of expansion as well as in different radial positions along the turbine blades can lead to obtaining more complex information about the distribution of the mentioned liquid phase parameters in the flow field of the steam turbine (ST).

Research of wet steam issues at Czech Technical University in Prague (CTU) has a long tradition. In this regard a measuring system with extinction probes has been developed which is regularly applied in turbine tests. Both the system and measurement methodology are continuously being improved. Currently the points of focus are:

- miniaturization of the extinction probe heads,

- automation and increasing the accuracy of data acquisition,

- improving data processing for more accurate results.

All these activities are related at some point to the interaction of the probe with the expanding wet steam. The measurement is invasive and as a result of the flow field interacting with the probe, the parameters measured in the probe head measuring space are different than the parameters at the same place without the probe. Particular positions and the process of this interference is the result of multiple phenomena, which can be suitably analysed through the use of CFD methods. The primary goal of the investigation of the interaction is to improve the measured data correction in the methodology used at CTU $[1,2]$. Current results of the CFD analyses also confirm the importance of its use for modifying the shape of the current instrumentation for low pressure (LP) steam and for designing new heads of the extinction probes developed for the research of high pressure (HP) wet steam.

This paper first summarizes the results from a new series of CFD simulations focused on the influence of the probe on the diameter of the droplets and steam wetness. Therefore the computational model of steam flow in the LP section of the steam turbine has recently been extended by a module of non-equilibrium condensation. This allowed for direct observation of the formation and development of fine droplets. For a description of the phase transition, classical homogeneous nucleation theory and Gyarmathy's model for droplets growth [3] were used. The actual polydisperse structure of the droplets is substituted by monodisperse in the CFD simulations. Subsequently there a conceptual design of a probe head for research of the HP steam is introduced, in which the knowledge obtained in the CFD simulations has been applied.

\section{Head of LP extinction probe}

Gradually a number of LP extinction probes for the measuring system at CTU with various shapes and dimensions have been developed. Cylindrical geometry has been used for the newly presented CFD analyses, as was the case in previous works [1,2]. The shape and basic dimension are shown in Figure 1 on the left. Measuring space of $20 \times 13 \mathrm{~mm}$ is in case of this probe cut into a cylinder of diameter $21 \mathrm{~mm}$. A light beam with a diameter of $4 \mathrm{~mm}$ (shown in yellow in the figure) goes through this space. The light is scattered on the liquid droplets passing through the beam, thus decreasing the light intensity. Measuring the change of the light intensity can be used to determine the dimensions of the droplets and their number (steam wetness) [4]. An important issue of the measurement using the optical probe is the influence of the probe interaction with the

Corresponding author: michal.kolovratnik@fs.cvut.cz 
flow field in the surroundings of the probe. Due to this interaction the wet steam parameters in the measuring space, particularly in its active measuring volume defined by the light beam are different from what they would have been in case the probe was not present. Therefore, a correction of the measurement results is necessary.

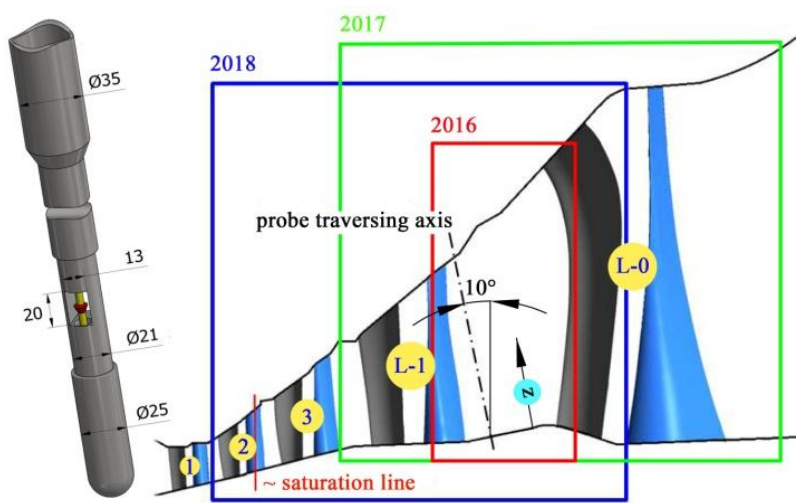

Fig. 1. The placement of the probe in the LP turbine

The right side of Figure 1 schematically shows a probe traversing axis in a 5 stage LP part of $1000 \mathrm{MW}$ ST with the measurement plane inclined $\sim 10^{\circ}$ from the radial plane. For data collection, the head of the extinction probe can be manually or automatically traversed to the desired position $\mathrm{z}$ along the blade height.

\section{CFD calculations}

Numerical research of the interaction of the optical probe with the expanding wet steam has been ongoing for several years. The numerical method used in this research has been subsequently improved both with respect to the computational model and properties of the working fluid. The extent of regions modelled over the years of our research is shown by the coloured borders in Figure 1 on the left (first red, then green and finally blue). In order to approach actual working fluid properties the previously used ideal gas has been substituted with water steam according to the IAPWS standard. The results, which were partly presented in [5], provided valuable knowledge about the impact of the optical probe on the flow field. The results also showed that for a deeper understanding of the interaction between the probe and the wet steam and of its impact on the liquid phase structure, it is necessary to analyse the formation and development of fine droplets in the turbine flow path. One of the methods used for this is numerical modelling of non-equilibrium condensation of the steam in the turbine.

\subsection{Non-equilibrium condensation modelling by ANSYS CFX}

The complex process of the formation and growth of fine droplets in expanding steam can be characterized by nucleation rate $J$ and droplets growth rate $d r / d t$. Nucleation rate $J$ defines the number of newly formed nuclei / droplets of critical diameter $r^{*}$ in unit time and unit volume of steam.

Non-equilibrium steam condensation is simulated in ANSYS CFX based on the Euler-Euler multiphase method. The fundamental system of equations for mass, momentum and energy balance is extended by simultaneously solved multi-phase models. These equations are then linked based on source terms. Such an extended system of equations is described in detail for example in [6]. The steam properties are described by the IAPWS standard, which also contains their formulations for the thermodynamically metastable region. Droplets are represented by a monodisperse system. By default ANSYS CFX uses the classical nucleation theory corrected by the non-isothermal factor to determine the nucleation rate $J$ and Gymarmathy's relation for the droplet growth rate $d r / d t$. This default settings can be adjusted and customized by users [7].

\subsection{The "2018" numerical model and explored cases}

For greater accuracy regarding the numerical simulation of the formation and growth of fine droplets and their interaction with the extinction probe, it would be best to model the entire flow passage of the 5 stage LP turbine section. However, such a complex approach would be too complicated and time-consuming. Therefore it was necessary to partly reduce the model.

The design calculation shows that at the inlet to the second stage of the LP turbine the steam is still superheated, i.e. single phase. The transition of the saturation line takes place in the rotor blades of this stage. Therefore exclusion of the first stage from the LP section model has minimal impact on the accuracy of the numerical simulation of the formation and growth of the fine droplets.

The modelled configuration considers the probe to be between the rotor blades of the second last stage (L-1) and the nozzle blades of the last stage (L-0) of the turbine (see Figure 1). Ending the model by the nozzle blades of L-0 will therefore provide sufficient accuracy for modelling the interaction of the fine droplets with the probe, assuming setting suitable boundary conditions for the model outlet.

Figure 1 shows the border of the final modelling region in blue of "three and half" stages which are included in the "2018" model. The model is divided into 8 calculation domains: S2, R2, S3, R3, S4, R4, S5 and PROBE shown in Figure 2.

The probe is placed in the PROBE domain, which is placed between R4 and S5 domains. The position of the probe is modelled in one of the measuring set-ups, particularly at the centre of the measuring space in $\mathrm{z}=385 \mathrm{~mm}$ from the hub of the rotor blades of the L-1 stage.

The calculation domain S2 consists only of a single inter-blade channel, other domains consist of several channels. The numbers of modelled channels are determined so that their tangential pitch is $\pi / 6$ in all cases. 


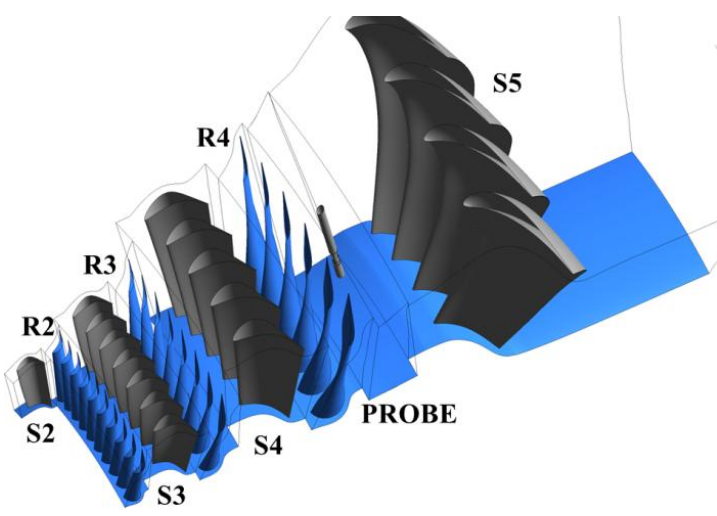

Fig. 2. Computation model of the variant with the extinction probe In this paper we consider and compare 2 variants. The first variant with the extinction probe (Var1) and the second one without the probe (Var0). The results of Var0 serve as a reference to assess the impact of the extinction probe in proximity and within the probe measuring space.

\subsection{Mesh generation}

The mesh of the calculation domains S2, R2, S3, R3, S4, $\mathrm{R} 4$, S5 are prepared in ANSYS TurboGrid while the mesh of the PROBE domain was created in ANSYS ICEM. Figures 3 and 4 show the surface mesh of the blade cascade and a detail of the mesh of the probe head. Var1 consists of 67.2 mil. hexagonal cells.

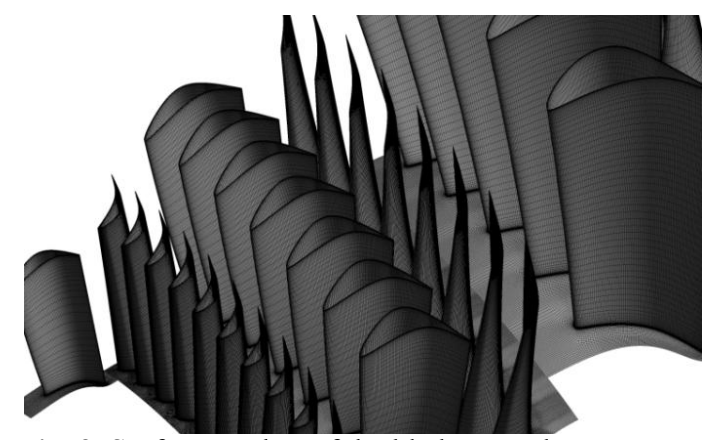

Fig. 3. Surface meshes of the blade cascades

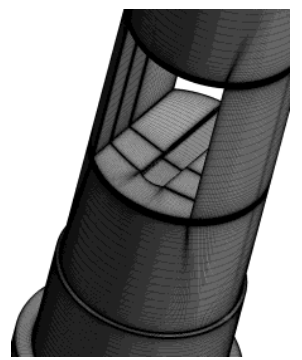

Fig. 4. Surface mesh detail of the extinction probe

\subsection{Boundary conditions and calculation set-up}

The inlet of the model uses a boundary condition of the type "pressure inlet" with profiles of the total pressure and temperature. The outlet of the model uses the condition "pressure outlet" with a static pressure profile. The profiles of total pressure, enthalpy and static pressure at the turbine inlet and outlet are exported from a CFD calculation of the entire LP turbine section (see Figure 5).

Together with the gas phase P1 only one liquid phase $\mathrm{P} 2$ is considered across all the computational domains.
Boundary conditions set for phase P2 at the inlet of the model are shown in Tab. 1.

Table 1. The boundary conditions for the liquid phase P2

\begin{tabular}{|l|c|}
\hline \multicolumn{1}{|c|}{ Boundary conditions } & Value \\
\hline Volume Fraction $(1)$ & 0 \\
\hline Specific Droplet Number $\left(\mathrm{m}^{-3}\right)$ & 0 \\
\hline
\end{tabular}
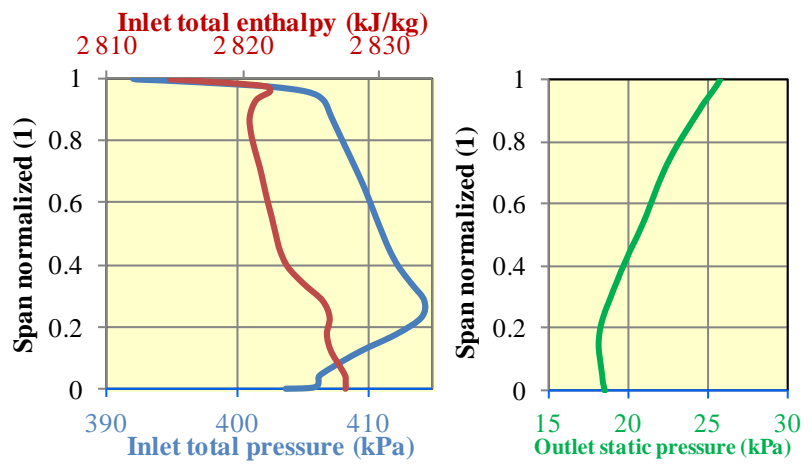

Fig. 5. Inlet total pressure and enthalpy and outlet static pressure distribution along the radial coordinate of the blade

For both phases the same two-equation turbulent model SST $\mathrm{k}-\omega$ is used. The rotor speed is set to $3000 \mathrm{rpm}$. Domain S2 is numerically connected to domain R2 by the model interface "Stage". Other domains are interconnected by the model interface "Frozen rotor". All calculations are made in ANSYS CFX 18.2.

\subsection{Results processing}

The primary goal of the CFD modelling is to analyse the distribution of wet steam parameters in the active cylindrical measuring volume, through which the light beam passes, in the measuring space of the probe (see section 2). Therefore first computation cells within the active cylindrical measuring space of the probe are selected. Final data processing is performed only on these selected cells using MATLAB.

\subsection{Discussion}

Figure 6 provides a comparison of the cumulative droplet count of fine droplets inside the active measuring space along the beam height for Var1 (red) and Var0 (blue). The data shows that the presence of the extinction probe decreases the overall number of fine droplets in the measuring space. In the case of Var1 $1.09 \cdot 10^{8}$ droplets were evaluated, while for Var0 there were $1.13 \cdot 10^{8}$ droplets.

Figure 7 shows both of the calculated variations of the distribution of the averaged diameter of the fine droplets along the measuring light beam. Clearly as the probe changes the flow field, there is also a change of the droplet diameter inside the active measuring volume. In the distribution for Var1, one interesting aspect is that near the bottom and upper boundaries of the measuring space the droplets have significantly lower diameters than in the central area. Near the bottom border this difference is more pronounced than near the upper one. Analyses of this issue revealed two phenomena that affect the droplet diameter in the probe measuring space. 


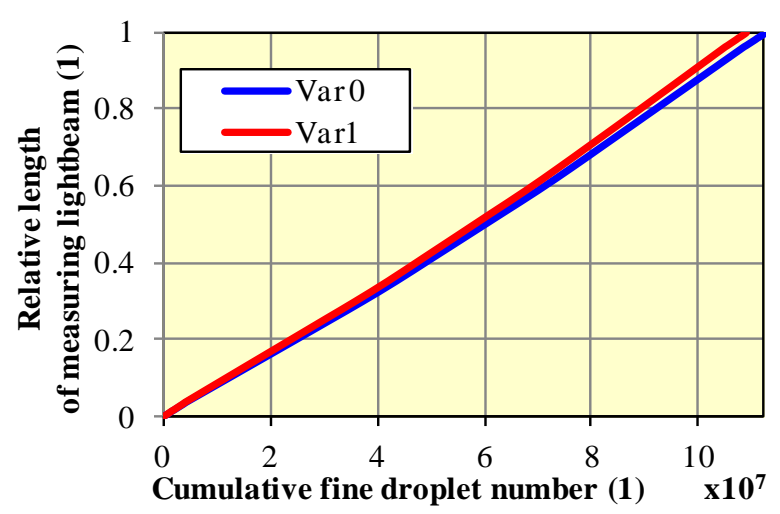

Fig. 6. Cumulative fine droplet number inside the active cylindrical measuring volume

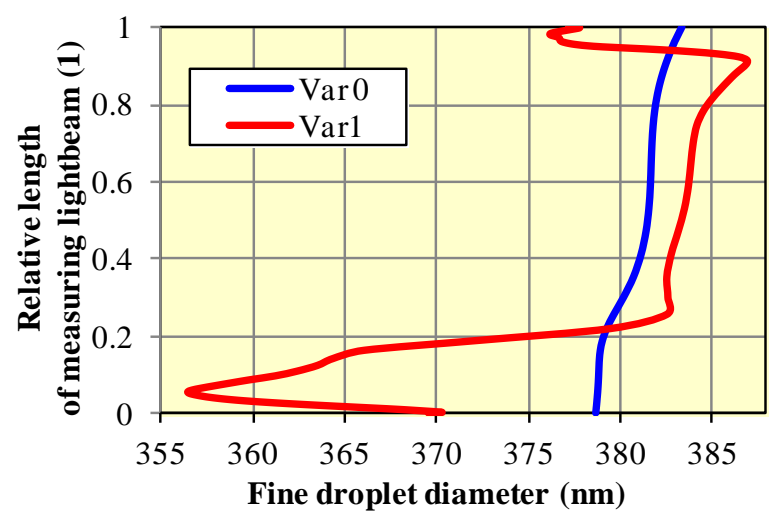

Fig. 7. Distribution of the average droplet diameter along the light beam

As seen in Figure 8, an intense backflow occurs behind the optical probe. This backflow strongly affects the area near the lower boundary wall of the measuring space. With the backflow of steam, fine droplets smaller than the droplets in the centre of the measuring space (unaffected by the backflow) are entering the measuring space. The droplet size is also affected by the local interphase mass exchange, i.e. evaporation of some of the fine droplets which occurs at the upper and lower boundary walls of the probe measuring space.

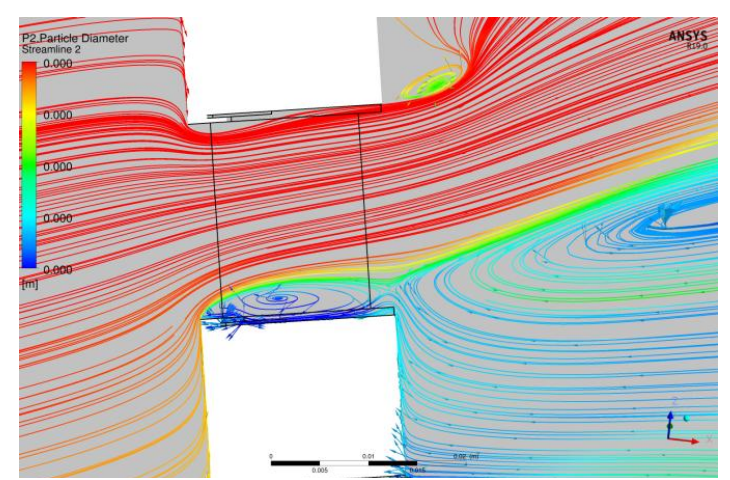

Fig. 8. Surface streamline colored by particle diameter of fluid phase $\mathrm{P} 2$

From Figure 9, it is clear that evaporation of the droplets takes place more intensely at the upper boundary wall than at the lower boundary wall.

The combination of these two phenomena causes a deformation of the fine droplet diameter distribution (see Figure 7), which can significantly complicate the correct interpretation of the measured extinction data.

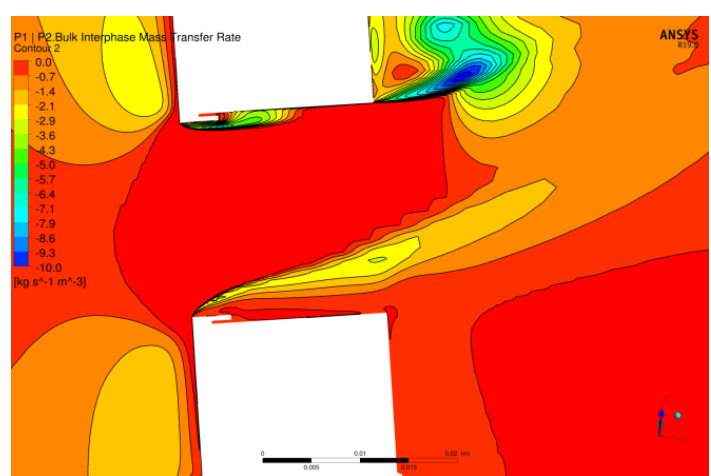

Fig. 9. Contours of $\mathrm{P} 1-\mathrm{P} 2$ interphase mass transfer rate

Figure 10 shows the steam wetness contours in the longitudinal cross-section of the probe measuring space. It is clear that steam wetness is significantly lower in the area near the upper and lower boundary walls of the measuring space than in the middle. As the steam flowing from the L-1 stage has a significant radial component of velocity, the impact on wetness distribution is much more pronounced in the area of the lower boundary wall than in the area at the upper boundary wall of the probe.

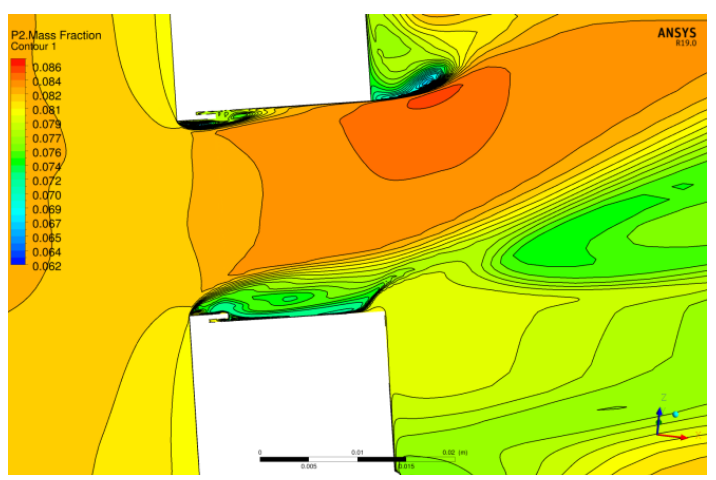

Fig. 10. Contours of steam wetness in the longitudinal crosssection of the probe

This fact is confirmed by the distribution of average steam wetness along the measuring beam for Var1 shown in Figure 11. This figure also shows the distribution of the average steam wetness for Var0, which corresponds to modelling of the real situation in the turbine without the probe. Unlike the distribution for Var1 (red line), the wetness distribution of VarO is practically constant. From a comparison of both, it can be assumed that the steam wetness measured by the extinction probe may be less than actual. However, it is

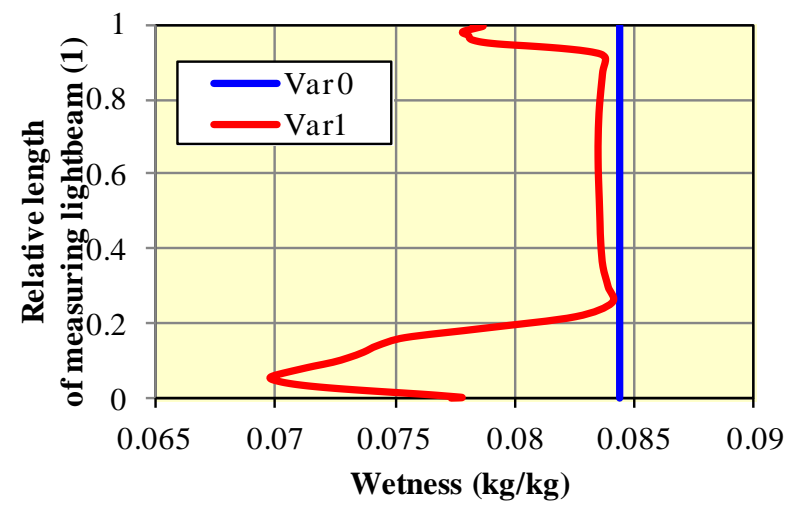

Fig. 11. Distribution of average steam wetness along the measuring beam for Var0 and Var1 
important to note that this applies only to the particular simulated traverse position, but not generally for all the probe traverse positions along the blade length.

Based on the CFD findings (here only briefly presented) of the flow-field and probe interaction, it can be summarized that the wet steam parameters in the probe's measurement space do change. Influence on the gaseous phase parameters and wetness has been proven earlier [1] and [2]. The new knowledge is about the influence on the liquid phase structure. This puts considerable demands on the methodology of correction of the measured data in terms of the influence of the flow-field by the probe, alternatively it extends the degree of uncertainty of the evaluated parameters. Formulation of a "universal" correction is hindered by the fact that the above-mentioned knowledge applies specifically to the simulated geometry of the flow passage, the probe traversing position and the given flow-field parameters. Therefore the results cannot be directly generalized. Modelling all measurement configurations is not a realistic option. It seems to be more effective to try to actively suppress the effect of the probe on the measured data by modifying the shape of the measuring head.

\section{Concept of the HP extinction probe head}

In recent years, increased attention has been paid to the phase transition during high-pressure steam expansion, which is currently associated with the HP part of steam turbines in PWR nuclear power plants. Theoretical analyses of these phenomena were also carried out at CTU and the measuring instrumentation of the liquid characteristics of the wet steam in HP steam turbines and moisture separators is under development.

Note: The necessary condition for the experimental research of liquid phase of $H P$ wet steam is to equip the high pressure part of the secondary circuit of the nuclear power plant with ports for insertion of the instrumentation. In the Czech Republic, such ports are not yet available. However, in future innovations and upgrades of the plants, it will be possible to implement these ports, most likely in the transfer pipe between the $H P$ turbine section and the moisture separator.

As there is absolutely no experimental data on the liquid phase structure in real HP steam turbines and separators, it was necessary to determine some data by numerical simulation. Therefore, available experimental data from HP nozzle experiments [8, 9] was analysed and corrections of phase transition models were determined. Subsequently, the calculation of the condensation in the conditions of the flow path of the 5 stage HP part of the wet steam turbine $1000 \mathrm{MW}$ was performed using the CTU statistical 2D model. Thus, basic information about the distribution of droplet dimensions and steam wetness at the outlet of the HP turbine was obtained. This made it possible to choose a measurement method and design a probe.

With respect to the current CTU approach to experimental research of the phase transition in LP wet steam, the optical method of multispectral light extinction was chosen from several other possible methods for further development. The basic design of the optical part of the HP extinction probe for the parameters at the HP steam turbine outlet confirmed the applicability of this method. However, due to the significantly higher optical density of HP wet steam in the area of the HP turbine outlet than what is typical in the experimentally explored LP turbines, it is not possible to use identical instrumentation. From the optical design of the probe, the actual length of the measuring space is in the order of millimetres. This is one to two orders lower than what is common with current LP instrumentation. Therefore the head of the extinction probe needs to be conceptually and structurally modified for HP application. In doing so, it is also necessary to respect the higher temperature and pressure loads of the probe elements.

Significant shortening of the measuring space means that the probe has a significantly higher influence on the flow field interaction on the measured parameters. Therefore, a number of proposed shapes for the HP extinction probe head have been tested through the use of the CFD methodology described in the previous part of this paper. On the left side of Figure 12 is an example of one of the tested probe designs to measure the structure of the wet steam liquid phase in the transfer pipeline between the HP turbine and the moisture separator. The considered diameter of this pipeline is 2 $\mathrm{m}$, and the average HP wet steam flow rate is about 50 $\mathrm{m} / \mathrm{s}$. The proposed location of the probe in the transfer pipeline is schematically shown on the right side of Figure 12.

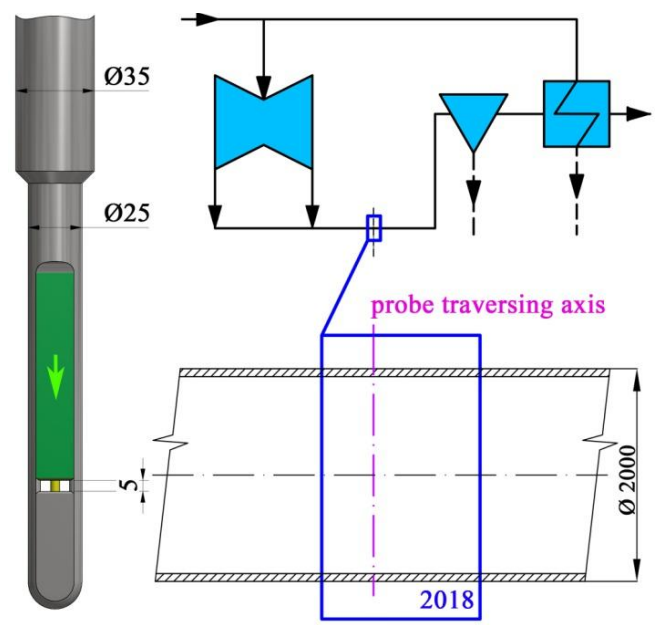

Fig. 12. Expected placement of the HP ext. probe in the piping During the design of the HP instrumentation, all the knowledge gained at CTU regarding the interaction between the probe and the flow field has been taken into account. The shape of the boundary surfaces of the measuring space has therefore been adjusted to minimize the influence of the flow. In view of the uncertainty in determining the spectrum of the droplet diameters by means of numerical simulations and in order to use the probe in other operating conditions, other particle sizes and, alternatively, for other pressures, a probe head with a variable geometry of the measuring space has been designed. By moving the part of the head (shown in 
green in Figure 12) we can change the length of the measuring space in the range of $1-5 \mathrm{~mm}$. For the purpose of further development of the instrumentation, an alternative design of the head with gradual measurement of extinction by several independent beams along the wet steam flow in the measuring space was proposed. This option should allow for a more detailed comparison of future HP experiments with numerical simulations. The technical feasibility and the optical arrangement of the HP extinction probes has been verified under laboratory conditions.

As an example of the CFD simulation results, Figure 13 shows the calculated distribution of droplet diameters and steam wetness in the measurement space of the test head of the HP extinction probe. It is intentionally presented with the head set with a very small height of the measuring space, particularly at $1 \mathrm{~mm}$. The uniform distribution of the monitored wet steam parameters in the transverse sections of the measuring space is apparent. To summarise, in terms of the interaction between the probe and the flow field, the proposed shape design of the HP probe head seems to be suitable for experiments in the conditions of the transfer pipeline.

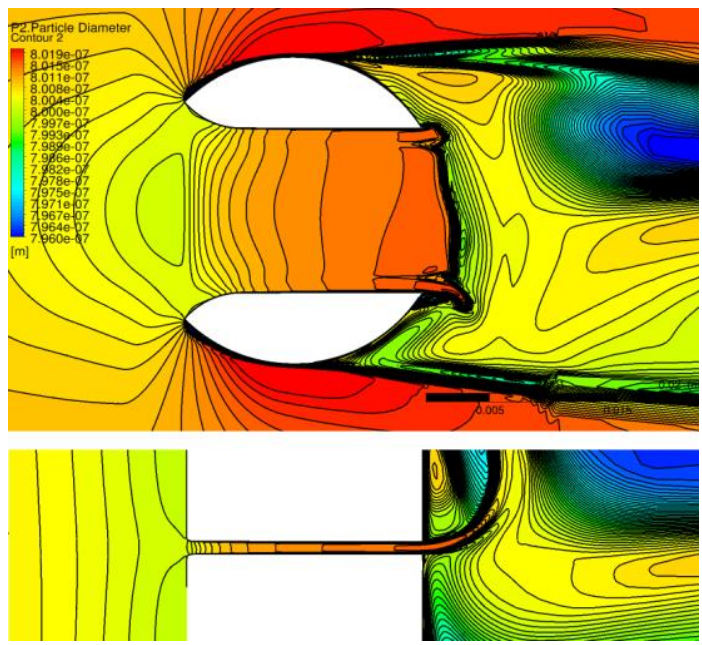

a) Distribution of droplet diameters in the measurement space of the HP extinction probe

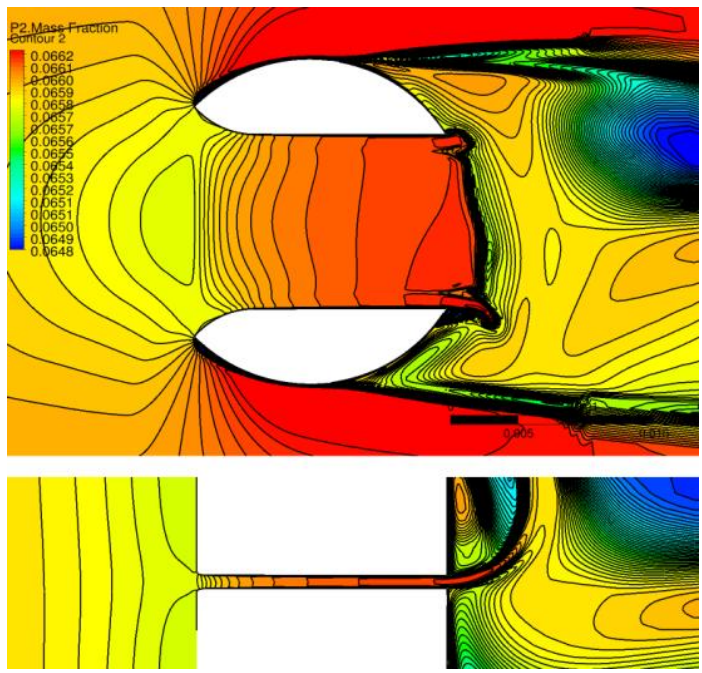

b) Contours of steam wetness in the measurement space of the HP extinction probe

Fig. 13. Example of the CFD simulation results

\section{Conclusions}

This paper briefly introduces the results obtained from a series of CDF analyses on the impact of the interaction between the LP extinction probe and the flow field of steam expanding in the turbine. These analyses were newly focused on the influence of the measured characteristics of the liquid phase.

Using these analyses, it has been proved that in the measuring space of the probe the dimensions, number of fine droplets and their distribution are different from that in the unaffected flow field. This raises new issues regarding possibilities of correcting the measured data or alleviating or eliminating these effects by changing the shape of the probe head. The incorporation of the acquired knowledge into the CTU methodology for extinction data correction will be the subject of further work.

The issue of suppressing the influence of the probe on the current field has been further investigated at CTU as part of a project focused on HP wet steam research. Conceptually, an HP extinction probe to investigate the structure of the liquid phase in HP expanding wet steam was designed and investigated using numerical simulations and partially through the use of laboratory tests. This concept can be implemented and deployed for research e.g. in nuclear power plants with PWR.

We gratefully acknowledge the support of grant no. 16-20123S of the Grant Agency of the Czech Republic.

\section{References}

1. M. Kolovratník, G. Jun, O. Bartoš, EPJ Web Conf. 143, 02052, (2017)

2. M. Kolovratník, G. Jun, O. Bartoš, EPJ Web Conf. 180, 02045, (2018)

3. G. Gyarmathy, Condensation in Flowing Steam. In Two-Phase Steam Flow in Turbines and Separators (Eds. Moore, M. J. and Sieverding, C. H.), ch. 3, Hemisphere, Washington, (1976)

4. C.F. Bohren, D.R. Huffman, Absorption and Scattering of Light by Small Particles, John Wiley \& Sons, Inc., USA, (1998)

5. G. Jun, M. Kolovratník, CFD analysis of optical probe influence on wet steam characteristic in LP steam turbine (in Czech). ANSYS Users meeting and conference, TechSoft Engineering, (2018)

6. J. Starzmann, M. Schatz, M.V. Casey, J.F. Mayer, F. Sieverding, ASME 2011 Turbo Expo, 7, GT201145672, (2011)

7. G. Jun, M. Kolovratník, Numerical simulation of steam condensation in Laval nozzle using ANSYS $C F X$. ANSYS Users meeting and conference, TechSoft Engineering, (2017)

8. F. Bakhtar, K. Zidi, Proc. IMechE, Part A, 203 (A3), (1989)

9. G. Gyarmathy, Proc. IMechE, Part A, 219 (A6), (2005) 